\title{
Gradhiva
}

Revue d'anthropologie et d'histoire des arts

19 | 2014

"L'Atlantique Noir » de Nancy Cunard. Negro Anthology 1931-1934

\section{Lettres de Claude McKay à Nancy Cunard}

Traduites et présentées par Anthony Mangeon

\section{Anthony Mangeon et Claude McKay}

\section{(2) OpenEdition}

\section{Journals}

Édition électronique

URL : http://journals.openedition.org/gradhiva/2812

DOI : 10.4000/gradhiva. 2812

ISSN : 1760-849X

Éditeur

Musée du quai Branly Jacques Chirac

Édition imprimée

Date de publication : 1 mars 2014

Pagination : 192-201

ISBN : 978-2-35744-073-9

ISSN : 0764-8928

\section{Référence électronique}

Anthony Mangeon et Claude McKay, "Lettres de Claude McKay à Nancy Cunard ", Gradhiva [En ligne] 19 | 2014, mis en ligne le 01 mars 2017, consulté le 20 avril 2019. URL : http:// journals.openedition.org/gradhiva/2812 ; DOI : 10.4000/gradhiva.2812 

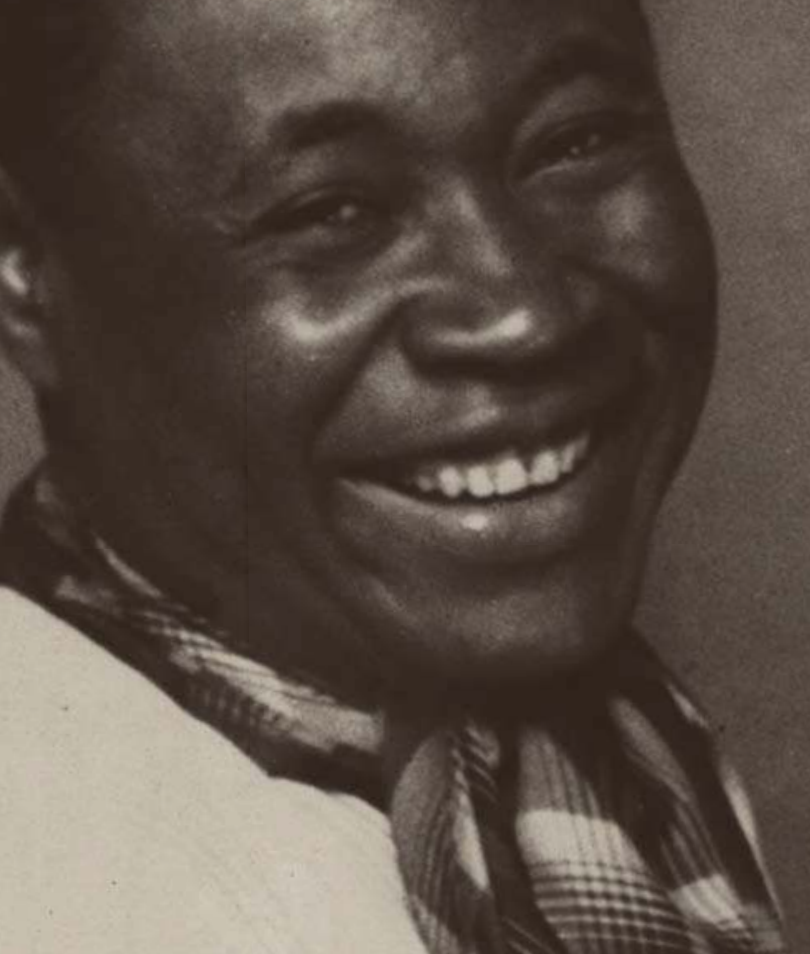

1 Null $\equiv$
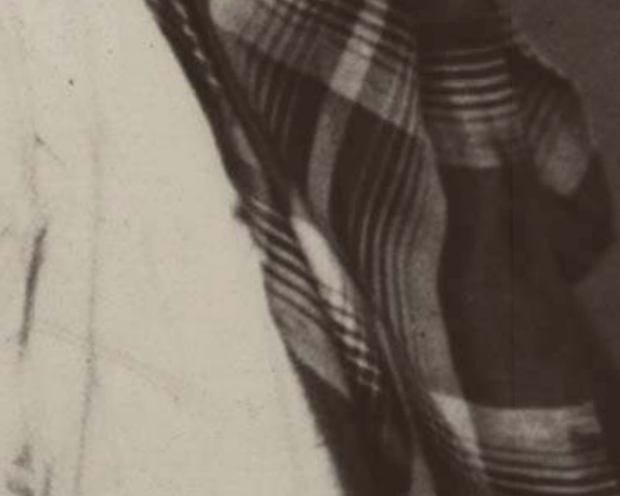

ণ্ণ

돈

몽

兘

19 


\title{
Lettres de Claude McKay à Nancy Cunard
}

\author{
Traduites et présentées par Anthony Mangeon
}

\section{La rencontre manquée de Nancy Cunard et de Claude McKay}

En 1931, Nancy Cunard, qui a désormais un vaste projet d'anthologie sur le monde noir, multiplie les rencontres et les correspondances à ce sujet. Son argumentaire ${ }^{\mathbf{1}}$ commence à circuler et, en décembre, elle reçoit enfin de Tanger une réponse du fameux écrivain jamaïcain Claude McKay (18891948). Celui-ci revient d'abord sur leur précédente «rencontre manquée», lorsqu'il faisait office de modèle, quelques années auparavant, pour l'artiste et écrivaine galloise Nina Hamnett (1890-1956), tandis que Nancy se trouvait «dans la pièce à côté». Et puisque cette dernière veut publier une photographie de lui avec la contribution qu'il promet de lui envoyer pour «l'anthologie nègre", qu'elle conserve, lui écrit-il, son portrait fait à la même époque par Berenice Abbott (nous le reproduisons ci-contre). Ne serait-ce point là une belle manière de rattraper le temps perdu et de donner enfin naissance à cette rencontre jadis avortée?

En 1934, pourtant, le nom de McKay ne figure point au sommaire de Negro Anthology. Et dans le dernier chapitre de son autobiographie, Un sacré bout de chemin (1940, traduction française de 1999), McKay revient en détail sur les divergences qui firent au final de cette rencontre épistolaire, entre lui et Cunard, un second rendez-vous manqué.

Tout avait pourtant bien commencé. Il lui écrit dans sa première lettre:

L'idée de votre livre m'enthousiasme, bien sûr, parce que je crois, à en juger par votre attitude et l'angle que vous avez choisi, si je les comprends bien, que vous produirez quelque chose de neuf et de très stimulant d'un point de vue artistique. Nous autres pauvres Nègres sommes, me semble-t-il, littéralement écrasés sous des tonnes de clichés rebattus et rassis dont nous gratifient nos amis, nos champions moraux - et dont nous nous gratifions nous-mêmes - sans jamais réussir à toucher quelqu'un. La plupart d'entre nous vivons dans la peur de nous-mêmes, des faits qui nous concernent. Nous sommes bien en peine de rendre la vérité artistique de nos propres vies telle que nous la connaissons et la ressentons; mais il serait inimaginable que vous vous laissiez entraver par les réactions sociales et raciales qui nous handicapent, parfois même inconsciemment. C'est pourquoi j'espère que ce que vous publierez sera une révélation et une inspiration.

Cette franche bénédiction, par l'un des papes de la littérature noire de l'époque, marque ainsi le début d'une passionnante correspondance qui s'étend sur plus d'un an, jusqu'en mars 1933, et dont une quinzaine de lettres - manuscrites ou tapuscrites - de McKay sont aujourd'hui conservées au fonds Nancy Cunard à l'université du Texas à Austin.
1. Voir François Buot, Nancy Cunard, Paris, Pauvert, 2008, p. 250-251. ci-contre

fig. 1

Berenice Abbott, Claude McKay, 1926. Getty Images (C) 2014 Berenice Abbott. 
2. "De la Jamaïque, Miss Cunard m'écrivit à nouveau qu'elle avait débarqué au paradis après son purgatoire new-yorkais où la presse l'avait prise pour cible quand on avait découvert qu'elle habitait à Harlem parmi les Nègres. [...] Pendant ce temps, j'étais au bord de la dépression en travaillant sur mon roman au Maroc, sans compter mes difficultés financières" (Claude McKay, Un sacré bout de chemin, traduction française de Michel Fabre, Marseille, André Dimanche, 1999, p. 358).
Ce qui frappe d'emblée, à la lecture de ces feuilles volantes, c'est l'extraordinaire disponibilité et sollicitude de McKay: on l'a souvent dépeint comme un écrivain ombrageux et susceptible, mais dès sa première réponse à Cunard il se révèle un être plein de bienveillance, conseillant la jeune femme dans ses voyages, multipliant pour elle les recommandations et mettant à sa disposition ses nombreuses relations, aux États-Unis et dans les Antilles, pour lui faciliter la tâche. Il lui envoie par ailleurs un exemplaire de son premier recueil américain, Harlem Shadows, tout en lui conseillant de se procurer, à l'occasion de son prochain voyage en Jamaïque, son tout premier recueil de vers, Songs of Jamaïca, qu'avec un certain dépit Nancy confondra avec le second, Constab Ballads (voir § 9). En retour, il sollicite deux livres, inaccessibles pour lui depuis le Maroc, et la remercie pour les nouveaux magazines ou journaux reçus.

Si de multiples considérations tournent ainsi autour de la littérature, et notamment des écrits respectifs de Cunard et de McKay (voir § 2, 10 et 12), leur correspondance prend également d'emblée une envergure plus large, et surtout politique: les thèmes récurrents en sont en effet la question raciale aux États-Unis et aux Antilles (ici § 7 et 8), la situation du communisme international, et son rôle dans le combat anticolonial et contre les exactions racistes des blancs. On sait combien la conception de l'anthologie Negro fut motivée par l'engagement de Cunard auprès des «Scottsboro Boys", ces jeunes Noirs injustement accusés du viol de deux femmes blanches en 1931. Lorsque à son retour à Harlem, en mai 1932, Nancy défraie la chronique des journaux new-yorkais en osant s'afficher à l'hôtel Grampion avec un Noir, son compagnon Henry Crowder, elle n'hésite pas à convoquer une conférence de presse et a tôt fait de transformer cette dernière en véritable tribune contre l'injuste procès qui a conduit à la condamnation à mort des neuf garçons un an plus tôt. L'écho de ce scandale est d'ailleurs parvenu jusqu'à McKay, depuis New York jusqu'à Tanger, et s'il est certainement édifié par tant d'audace et de tempérament, l'écrivain ne la met pas moins en garde contre les turpitudes à craindre de la part des services secrets britanniques (§ 1 et 2$)$.

La correspondance prend de fait, au fil des mois, une tournure plus sombre, et par là même un peu répétitive: car s'il se refuse à toute paranoïa (§ 2), McKay n'en est pas moins profondément affecté par le vol, dans sa petite maison marocaine, de tous ses papiers (carte d'identité française, passeport britannique) et du peu d'argent qu'il lui reste durant l'été 1932 (lettre du 31 août). Cet épisode le plonge, de son propre aveu, dans un certain état dépressif ${ }^{2}$. II connaît de surcroît d'importants problèmes de santé (il souffre notamment d'hypertension et de lombalgies chroniques, douloureux héritage de ses années marseillaises, quand il travaillait comme docker), et ces soucis sont constamment aggravés par des difficultés financières. Ne vivant en effet que de sa plume, il ne peut se permettre d'écrire pour rien: l'écrivain ne cesse ainsi de reporter, de mois en mois, le texte qu'il a promis à Nancy, lui parlant fréquemment de ses difficultés à boucler son nouveau roman, Banana Bottom, une «saga paysanne» (lettre du 18 septembre 1932) située dans la paroisse de Sainte-Anne, en Jamaïque. II attend de fait beaucoup de la publication de ce roman en Angleterre et de sa reprise en feuilleton par les éditions Rieder, qui avaient en 1931 publié une traduction 
de Banjo et fait paraître quelques poèmes de McKay dans la revue Europe. Mais Nancy comprend mal le rôle que l'écrivain lui demande de jouer: empressée à jouer les entremetteuses, alors que McKay lui demandait simplement de déposer pour lui son manuscrit chez Rieder, elle lui aliène quelque peu la sympathie de l'éditeur français, et l'écrivain jamaïcain lui en fera assez vertement le reproche. Ce ne sera malheureusement pas le dernier car lorsque, le 12 janvier 1933, McKay envoie son article et demande si l'anthologue a prévu une rémunération pour ses divers contributeurs, la réponse négative de Cunard ne tarde pas à l'offusquer. Certes, celle-ci l'avait auparavant dépanné en lui envoyant, dans l'urgence, un mandat postal (lettre du 29 septembre 1932), mais c'était là un geste amical, tout comme les livres reçus ou échangés. Si McKay se dit «romantique» au sujet de l'art (voir l'extrait de la lettre du 29 septembre 1932), au point de prendre ses distances avec les théories de l'engagement et du réalisme prolétarien, son romantisme, prend-il soin de préciser, est «bien différent de celui qui anime tous ces braves gens qui exigent et attendent des artistes qu'ils écrivent, chantent, jouent la comédie ou se produisent sous toutes les formes possibles, gratuitement et charitablement pour la bonne cause, alors qu'il ne leur viendrait pas même à l'esprit de faire travailler pour rien un charpentier, un traiteur ou tout autre artisan ou travailleur manuel» (lettre du 25 janvier 1933). Dans son autobiographie de 1940, il brocardera à nouveau chez Cunard cette attitude « dépassée, aristocratique et très britannique [qui considère] que les artistes doivent produire - à l'intention des gens nobles et riches - pour la gloire et non pour être payés ${ }^{3}$. Son refus de céder pour rien ses droits d'auteur et l'interdiction qu'il lui intime de publier son texte (un récit de son séjour à Londres intitulé «Up to date») rendront évidemment furieuse la jeune éditrice, et cette riche correspondance s'arrêtera brutalement.

Sans doute y avait-il déjà quelques éléments de fracture dans les lettres échangées. Ainsi, le détachement de McKay vis-à-vis de l'orthodoxie communiste (lettres du 29 septembre et du 15 octobre 1932) ou ses jugements profondément négatifs à l'égard d'autres écrivains et penseurs caribéens - notamment Marcus Garvey et son disciple Eric Walrond (lettre du 18 septembre 1932), avec qui Cunard eut certainement une liaison durant le printemps $1931^{4}$ - sonnent comme autant de confidences hautaines qui devaient altérer la sympathie qu'éprouvait pour lui cette jeune femme par ailleurs profondément éprise, à l'époque, de la faconde révolutionnaire de la presse noire. Dans Negro Anthology, elle écrira en effet de McKay que «son peuple [les personnages de ses romans] et lui ont la même conscience de race erronée; ils forment des cercles fermés, ils sont ombrageux ${ }^{5}$ ». Mais en considérant l'ampleur des vues, et la densité des propos échangés durant cette année de confidences partagées, le lecteur contemporain et curieux des coulisses des mouvements nègres dans l'entre-deux-guerres ne peut que regretter qu'en entrant avec fracas dans I'histoire littéraire, Cunard et McKay aient dans le même temps raté leur rendez-vous épistolaire avec la postérité.
3. Claude McKay, Un sacré bout de chemin, op.cit., p. 359.

4. Écrivain et journaliste né en Guyane Britannique, Eric Walrond (1898-1956) rédigeait des chroniques pour la presse noire (The Negro World, Opportunity) mais cessa de publier après son départ pour l'Angleterre, en 1928. Sur sa liaison avec Cunard, voir la biographie de cette dernière par François Buot, op.cit.

5. Nancy Cunard, "Harlem reviewed ", dans Hugh Ford (éd.), Negro: An Anthology, édition abrégée, New YorkLondres, Continuum, 2002, p. 53; cité par McKay lui-même dans son autobiographie, op.cit., p. 360 . 
6. Hôtel situé à Harlem, dans lequel Nancy Cunard s'installa au printemps 1931 avec son compagnon afro-américain, Henry Crowder. À la suite du Daily Mirror, qui annonça dans son édition du 2 mai 1931 que «l'héritière de la célèbre ligne transatlantique " était descendue à Harlem pour y suivre le chanteur Paul Robeson (1898-1976), la presse américaine et britannique fit ses choux gras de cette rumeur médiatique.

7. Arturo Schomburg (1874-1938) était un écrivain et historien afro-américain, d'origine portoricaine, qui établit une importante collection bibliophile sur le monde noir, devenue la bibliothèque Schomburg à New York.

8. Black Man and White Ladyship, 1931.

9. Frank Harris (1856-1931) était un écrivain et journaliste américain d'origine irlandaise qui dirigea d'importants périodiques, en Angleterre et aux États-Unis (The Saturday Review, Pearson's Magazine), et qui se rendit par ailleurs célèbre pour ses mémoires (My Life and Loves), publiés en plusieurs volumes (1922-1927), dans lesquels il abordait sans détour ses aventures sexuelles tout en s'attribuant un rôle d'éminence grise dans de nombreux événements historiques.

10. La publication de son roman L'Amant de Lady Chatterley (1928) valut à l'écrivain et poète anglais David Herbert Lawrence (1885-1930) d'être poursuivi par les autorités américaines et britanniques pour propagande licencieuse; les services postaux furent alors chargés de saisir ses ouvrages et interceptèrent la même année le tapuscrit de ses Pansies Poems.
11. Écrivain roumain francophone (1884-1935), aux sympathies communistes, auteur d'un cycle romanesque Les Récits d'Adrien Zograffi (1923-1926) et d'une critique informée des dérives dictatoriales de la Russie soviétique (Vers l'autre flamme, 1929).

12. Écrivain français (1873-1935), auteur d'un fameux récit sur la première guerre mondiale (Le Feu, prix Goncourt 1916), grand admirateur de la révolution russe et partisan d'une "littérature prolétarienne».

13. Allusion à une formule méprisante que Jan Smuts (1870-1950), militaire et homme politique sud-africain, avait eue à l'égard des populations africaines, et qui sera reprise et critiquée par le publiciste et écrivain afro-américain William Edward Burghardt Du Bois dans un fameux éditorial de la revue The Crisis («Patient Asses»), en mars 1930 (NdT).

\section{Chère mademoiselle Cunard,}

Vos lettres et les journaux jamaïcains m'ont empli de nostalgie... Avant de les avoir sous la main (la distance entre la lointaine Jamaïque et Tanger a pris du temps), j'étais déjà informé de vos divers déplacements par voie de presse. D'abord, les journaux franco-marocains se sont fait l'écho du "scandale du Grampion'6", puis le New York Herald (dans son édition parisienne) a rapporté, comme une nouvelle sans intérêt, votre départ de Harlem pour la Jamaïque. Joy m'a également écrit pour me raconter l'aprèsmidi passé en votre compagnie et celle du gros Schomburg ${ }^{7}$.

J'ai été chagriné par toutes ces calomnies - d'autant qu'elles intervenaient avant que vous ayez pu collecter tous vos matériaux et lancer votre anthologie. Je ne m'explique pas la manière dont vous avez été traitée à Cuba - j'y vois l'œuvre néfaste des services secrets britanniques, plus encore que de la presse, et pourtant je n'ai pas encore, en dépit des avanies subies, développé de délire de persécution... Néanmoins, je pense que vous devriez vous méfier d'eux et rester sur vos gardes, dans l'élaboration de votre anthologie et dans vos divers voyages, car ils pourraient bien tenter de vous nuire et de mettre en péril votre travail. Votre pamphlet sur L'Homme noir et la femme blanche ${ }^{8}$ a dû rendre furieux toutes celles et ceux qui, du haut de l'échelle sociale, luttent bec et ongles pour maintenir l'impérialisme britannique dans son prestige et sa superbe anglo-saxonne. Quand je vis Frank Harris pour la dernière fois en 1927, il m'expliqua qu'on lui interdisait de rentrer en Angleterre et que des agents britanniques étaient à la manœuvre pour le faire expulser de France comme un indésirable à cause de ses Confessions $^{9}$. Je suppose que vous êtes au courant du comportement tyrannique des autorités de la Poste britannique à propos des poèmes de D.H. Lawrence ${ }^{10}$... Un cas de plus. Quand je me trouvais en Angleterre, juste après la signature de l'armistice, j'avais un ami anglo-allemand qui faisait office d'éditeur adjoint et de caricaturiste pour un petit journal anarchiste appelé L'Éperon. Un vieil aristocrate anglais qui vivait en Italie avait coutume de nous envoyer des articles et de l'argent. J'ai lu certaines de ses lettres - elles n'étaient vraiment pas dangereuses, plutôt l'œuvre d'un idéaliste un peu anar. Cependant, après le coup d'État fasciste, il dut quitter l'Italie pour émigrer au Mexique, puis au Japon et dans d'autres pays. Et il écrivit à mon ami que, partout où il se trouvait, la police secrète l'espionnait... Il existe apparemment une police internationale dont la vocation est de mener la vie dure aux communistes et à leurs simples compagnons de route... Voici quelque temps, Panaït Istrati ${ }^{\mathbf{1 1}}$ s'est vu interdire l'entrée en Égypte, et il est récemment arrivé la même chose à Henri Barbusse ${ }^{12}$ en Angleterre.

En vous intéressant à cette partie de l'humanité que le brillant général Smuts appelle «les culs patients ${ }^{13}$ », vous rencontrerez, je pense, beaucoup d'opposition et d'intrigues, surtout si votre projet dépasse le simple domaine artistique. Étant donné que cette masse constitue la dernière réserve docile 
parmi les exploités, ses exploiteurs entendent bien la maintenir perpétuellement dans cet état, et ils prennent ombrage de tout ce qui peut nourrir chez eux un autre esprit que celui de «culs patients».

Visiblement, elle fut à vomir, votre visite aux États-Unis! J'ai l'impression que vous n'avez pu faire tout ce que vous y envisagiez - un voyage dans le Sud, et un autre à Washington chez les Noirs qui se prennent pour des aristocrates. II eût peut-être été préférable de vous arracher à la petite troupe qui gravite autour de Carl Van Vechten ${ }^{\mathbf{1 4}}$. Le connaissez-vous? II pourrait bien être has been. Je n'entends plus beaucoup parler de lui à présent. Je n'ai pas essayé de vous donner mes contacts à New York étant donné que vous vous y trouviez déjà, que vous aviez écrit pour le magazine The Crisis et que vous aviez un ami afro-américain. James Weldon Johnson (l'ancien secrétaire de la NAACP ${ }^{15}$ ) est un homme vraiment très bien, très différent de Du Bois ${ }^{\mathbf{1 6}}$ et de Walter White ${ }^{\mathbf{1 7}}$, et je suis sûr qu'il vous aurait comprise et aidée. De surcroît il a de bons contacts à Haïti et à Cuba, dommage que vous n'ayez pu visiter ces deux îles. Mais vous devez connaître James Weldon Johnson et sa charmante épouse ${ }^{\mathbf{1 8}}$.

Votre débâcle à New York m'affecte car je suis convaincu qu'en dépit de ses défauts et du snobisme des Harlémites, un mouvement propre aux Noirs - qu'il soit artistique ou politique - ne pourra émerger qu'à New York. Les Antilles ne sont pas à la hauteur, même si, de mon point de vue, leur contribution sera grande en raison de leur contexte social. Et comme votre livre diffère tellement, dans son esprit comme dans son plan, de tout ce qui s'est fait jusque-là (car, fort heureusement, vous vous tenez à l'écart de tous les groupes qui s'intéressent au Noir), j'ai pensé qu'il pourrait bien devenir, après sa publication, le point de départ d'une nouvelle expression. C'est en considérant ainsi votre travail que je me sens contrarié par les frayeurs et les scandales que répandent les journaux, et qui pourraient vous déprimer et vous décourager et peut-être réduire les chances d'une bonne réception de votre œuvre parmi les critiques et les gens qui comptent dans le monde de l'art.

Je suis heureux que la Jamaïque vous ait plu, désolé que Cuba ait été un fiasco (Langston Hughes ${ }^{19}$ avait passé du bon temps à La Havane parmi les Noirs cubains), et aussi que vous n'ayez pu visiter Trinité, et tout particulièrement Haïti. J'ai bien ri en lisant le récit de l'accueil dont on vous a gratifiée, et ces discours sur «le martyr» (cela m'a rappelé mon séjour en Russie, où j'avais été reçu en véritable consul20!), mais la vision de Garvey ${ }^{21}$ avec son sourire béat et ses programmes «Que Dieu vienne en aide à notre roi » me remplissent de tristesse et de colère $\mathbf{2 2}$... Bien évidemment, il est bien content d'être redevenu un petit Jamaïcain après avoir tout simplement ruiné sa chance à force d'enchaîner les erreurs, et en dépit des avertissements et des conseils reçus, de devenir un grand leader mondial. Ceux qui ont assisté à son procès disent qu'avec sa suffisance démesurée et ignare, en tant que «président provisoire de l'Afrique» entouré de ses chevaliers, seigneurs, dames et ducs tout droit sortis de Liberty Hall, il a littéralement privé ses avocats de toute ligne possible de défense et s'est précipité de lui-même en prison. C'est assez méchant de penser cela après coup, mais peut-être se trouvait-il fort aise de se voir ainsi mis sous les verrous car la chose qu'il avait créée le dépassait de beaucoup, comme il devait en avoir
14. Écrivain et photographe américain (1880-1964), proche des écrivains de la Renaissance de Harlem et auteur d'un roman (Nigger Heaven, 1926) centré sur la vie des Noirs à New York.

15. Poète, romancier et diplomate afro-américain, James Weldon Johnson (1871-1938) aborda la question des identités simulées (les «Noirs» suffisamment clairs de peau pour se faire passer pour des "Blancs») dans son Autobiographie d'un ancien homme de couleur (1912) avant de devenir secrétaire exécutif de la National Association for the Advancement of Colored People (NAACP) jusqu'en 1931.

16. William Edward Burghardt Du Bois (18681963) est l'une des grandes figures afro-américaines du $x x^{e}$ siècle: tour à tour universitaire (historien, sociologue), publiciste, écrivain, il fut l'un des fondateurs de la NAACP dont il dirigea la revue The Crisis jusqu'en 1934.

17. Walter White (1893-1955) était un journaliste et romancier afro-américain suffisamment clair de peau pour passer pour blanc, et qui fit usage de ce "privilège" pour observer de nombreux lynchages et les dénoncer dans la presse ou devant les tribunaux. Il était également membre de la NAACP.

18. Sur tout cela, voir également l'autobiographie de Claude McKay, op.cit., p. 121-122 (NdT).
19. Poète, romancier et journaliste afro-américain (1902-1967), auteur majeur de la Renaissance de Harlem.

20. Allusion au séjour qu'effectua McKay en Russie soviétique, de l'hiver 1922 au printemps 1923, et où il assista notamment au quatrième congrès de l'Internationale communiste, en compagnie de nombreux autres écrivains communistes.

21. Marcus Mosiah Garvey (1887-1940) était un influent activiste et publiciste jamaïcain, devenu célèbre pour avoir créé I'Universal Negro Improvement Association en 1916, ainsi qu'un journal, The Negro World. Après avoir été emprisonné aux États-Unis pour fraude postale, il fut déporté vers son pays d'origine et mourut finalement en Angleterre.

22. Bien sûr, je ne nierai pas que ce que fait Garvey en Jamaïque est utile, mais c'est tellement insignifiant en comparaison avec les chances qu'il a gâchées aux États-Unis. Étant donné que vous n'avez pu vous rendre en Haïti, si vous n'avez pas de contact là-bas, vous pourriez obtenir de bonnes informations auprès de William Seabrook, l'auteur de Magic Island. Merci pour les photos. Dommage que vous n'ayez pu en avoir une de mon père. J'ai maintes fois demandé à mon frère de m'en envoyer une ( $\mathrm{NdA})$. 
23. Institut de formation technique et agricole créé par le leader afro-américain Booker T. Washington (1856-1915), autodidacte afro-américain qui avait connu l'esclavage et jugeait que les Noirs devaient d'abord s'élever économiquement et matériellement pour faire valoir ensuite leurs droits politiques. II fut vivement critiqué par Du Bois pour cette position complaisante à l'égard du racisme blanc, et pour s'être attaché au seul enseignement professionnel au détriment d'un accès plus large des Noirs à l'enseignement supérieur. bien conscience. Le gouvernement britannique a finalement placé Garvey sur une petite île, et tout le monde - lui autant qu'eux - y trouva son compte: il y peut encore accéder aux plus hauts honneurs - devenir par exemple un membre du Conseil législatif.

Les milliers de dollars qu'il a collectés, il les a gaspillés alors qu'ils auraient pu servir à financer un bon théâtre, un hôtel ou un centre social pour les Noirs. Et ce sont les Afro-Américains qui ont perdu le plus d'argent car, en doux rêveurs, ils ont poursuivi la chimère d'une utopie politique pour les Noirs et ce sont surtout eux qui ont acheté des parts dans l'entreprise de la Black Star Line, tandis que les Antillais se contentaient d'en faire la publicité. À cause de Garvey, le fossé entre les groupes noirs américains et antillais aux États-Unis est plus large que jamais. Garvey n'a fait qu'accentuer entre eux les jalousies, rivalités et discordes - et c'est si stupide! II n'a cessé d'insister sur les différences entre Noirs et mulâtres, alors que ces dernières ont moins de réalité aux États-Unis qu'aux Antilles, où elles constituent en effet la base des différences de classe. Les Noirs américains peuvent bien jouer la gamme aristocratique des différences de couleur, ces nuances ne seront pas reconnues par le gouvernement ni par les Blancs et n'auront donc aucune influence sociale comme c'est le cas aux Antilles et en Afrique du Sud. Qu'ils soient foncés ou clairs de peau, les Afro-Américains constituent un seul bloc social et politique. Je ne peux m'étendre davantage là-dessus dans une lettre, mais Américains et Antillais ont des torts partagés dans la situation qui les oppose actuellement. L'Antillais, parce qu'il a joui chez lui d'une plus grande liberté sociale, est souvent et sans justification aucune arrogant avec l'Américain, et c'est tout particulièrement vrai des Antillais britanniques. Je les ai souvent entendus proférer des stupidités sur la qualité de la justice britannique envers les autochtones, à partir de leur seule petite expérience d'Antillais. D'un autre côté le Noir américain brocarde la grossièreté de la plupart des immigrants antillais, de leurs vêtements, leur accent et leur esprit britannique quand c'est le cas.

En Amérique j'ai toujours préféré les Noirs américains aux Antillais qui, en toute occasion, manifestent un esprit de caste trop marqué à mon goût; quand je vivais là-bas, je côtoyais des Américains plutôt que des Antillais. Bien sûr, j'ai pu dès le départ prendre mes distances. C'est en effet dans le Sud que j'ai débarqué à l'occasion de mon premier voyage en Amérique et, après six mois passés à Tuskegee ${ }^{23}$, je me suis rendu dans le Middle West où je suis resté deux ans avant de rejoindre finalement New York. Les Antillais sont plus appréciés par les Afro-Américains dans le Sud et dans l'Ouest que dans l'Est. Et puis j'étais étudiant, mais quand j'ai pris un travail de domestique je me suis fait d'excellents camarades - bizarrement, je ne me suis disputé qu'avec d'autres Antillais. Les Noirs antillais sont solides et semblent bien plus fiables que les Américains en règle générale, mais j'aime l'esprit enjoué et fêtard des Américains.

Merci beaucoup pour les livres. Je me demande si en définitive vous avez trouvé les bons poèmes. Mon premier recueil publié s'intitulait Songs of Jamaïca. C'est celui où je figure au recto dans mon uniforme de gendarme, à ne pas confondre avec mon second recueil, qui lui s'intitulait Constab Ballads mais que je trouve moins bon. Mon premier recueil contenait mes 
anciens poèmes en dialecte, mais je crains que les publicités avec «le gendarme" en bandeau ne vous aient fait acheter le mauvais recueil. Ce dernier contient des poèmes écrits pour la circonstance (une seconde publication) alors que mes premiers vers manifestaient une véritable spontanéité lyrique à propos de choses aussi diverses que les collines de Clarendon, les paysans, la vie agricole et celle des marchés... Mais merci pour le mal que vous vous êtes donné et pour vous en être souvenu en dépit de tous vos soucis et des si nombreuses choses auxquelles vous deviez penser. Je suis très heureux que vous ayez trouvé L'Histoire de la Jamaïque.

Mon prochain livre traitera à loisir du phénomène de l'obeah. C'était et c'est encore une réalité profondément inscrite dans la culture jamaïcaine. Quand je vivais à Clarendon, il était rare qu'une famille paysanne n'adhère pas ou ne se livre pas à de telles pratiques. Dans notre village, il y avait seulement trois exceptions et, dans les villes comme Kingston, c'était un culte florissant. Ceux qui ne consultent pas l'obeahman n'en vont pas moins voir les clairvoyants. C'est bizarre que mon frère ne vous en ait pas parlé. Je me souviens qu'enfant, il avait l'habitude de nous sermonner à ce sujet et qu'il s'évertuait à convaincre ses amis aisés, dans le monde paysan, que le culte obeah n'était qu'une chimère. Mais je suis sûr que c'était en vain.

Comme il a grossi! II s'est toujours très bien porté mais sans atteindre ces proportions. C'est un héritage de ma mère et je me bats quotidiennement contre cette propension à s'arrondir. Notre père (j'espère que vous l'avez vu) est aussi élancé qu'un lévrier et ses frères étaient tous maigres. II a plus de 90 ans maintenant.

Bon, je suis désolé que ma contribution ne soit pas encore prête, mais je vous promets de vous l'envoyer dans les temps. Je vous ai dit que je m'y mettrai dès que mon prochain livre sera pour moitié achevé et expédié à mon éditeur. J'espérais que cela me vaudrait une autre avance, me permettrait de prendre un peu de repos et de faire ce que je vous ai promis dans l'intervalle. Je souffre d'hypertension depuis quelques années et je ne peux me concentrer que sur une seule chose à la fois. Mais, hélas! Gingertown a été un inquiétant fiasco. II ne s'en est vendu que 1100 exemplaires - c'està-dire pas suffisamment pour couvrir le quart de l'avance qui m'avait été consentie, et les éditeurs refusent à présent de me rémunérer davantage. Ils m'ont également irrité en négligeant de répondre à mes lettres, au point que j'ai dû gaspiller de l'argent pour leur envoyer un télégramme à l'étranger. Je n'avais d'autre choix que de continuer à bûcher ferme, mais le pire c'est que mon ami Max Eastman ${ }^{\mathbf{2 4}}$, qui d'habitude défend mes intérêts à New York, se trouve actuellement quelque part à Constantinople pour rendre visite à Trotski. Je n'avais donc personne pour reprendre contact avec les éditeurs. J'ai terminé et je leur ai envoyé trois parties du livre. Je suis toujours sur la quatrième et j'espère l'achever à la fin du mois. Entre-temps j'ai écrit à quelqu'un à New York pour lui demander d'essayer de vendre mon texte à un autre éditeur - en dépit du contrat avec Harper. II doit bien se trouver un éditeur disposé à me payer une avance suffisante pour me permettre de rembourser Harper! Je ne sais si ma lettre est parvenue à bon port ni si ce plan est réalisable - on dit que le marché du livre traverse une telle crise... J'ai aussi demandé à Rieder, mon éditeur français ${ }^{\mathbf{2 5}}$, de m'acheter d'emblée
24. Écrivain et journaliste américain (1883-1969) d'obédience socialiste. Devenu ami de Léon Trotski, il séjourna en Union soviétique de 1923 à 1925 et publia à son retour un essai très critique sur l'évolution du régime stalinien.

25. Maison d'édition française fondée en 1913, soutien des écrivains de gauche et de la revue Europe, qui fusionna en 1939 avec d'autres éditeurs parisiens (Félix Alcan, Ernest Leroux) pour fonder les Presses Universitaires de France. 
26. Allusion à la revue Europe, créée en 1923 par Romain Rolland (1866-1944), écrivain pacifiste et prix Nobel de littérature.

27. Paulette Nardal (18961985) était une journaliste et essayiste martiniquaise qui fonda en 1931 la Revue du monde noir, dont les six numéros bilingues (français-anglais, 1931-1932) contribuèrent à faire connaître les écrivains afro-américains en France et à lancer le mouvement francophone de la négritude. mon nouveau manuscrit pour le publier en feuilleton dans leur magazine ${ }^{\mathbf{2 6}}$, et j'attends leur réponse. Cela ne constituerait pas un non-respect des droits d'auteur aux États-Unis et, quand bien même, je pourrais toujours demander que cette clause soit levée.

Je suis en train d'écrire le dernier chapitre et dès qu'il sera achevé et qu'une solution sera, d'une manière ou d'une autre, trouvée pour ce manuscrit, je me remuerai les méninges pour votre article.

Qu'est-ce que l'Excelsior? Un hôtel ? Quand je m'y trouvais, il n'y avait même pas d'hôtel à Chapelton et la pension de Mary Pin's était si pauvre que rares étaient les touristes qui s'aventuraient le long de la vallée du Rio Minho. Je suppose que le chemin de fer et le commerce de la banane ont dynamisé Frankfield. Mon livre parle de toute cette région et de la paroisse voisine, Sainte-Anne, qu'on appelle «le jardin de la Jamaïque». Vous serez donc bientôt en mesure de juger si je m'en souviens bien et si les scènes de ma composition en restituent bien l'ambiance. Oui, les gens de l'arrière-pays sont merveilleux. Ils n'ont juste aucune idée des difficiles exigences morales et pratiques de l'Europe et de l'Amérique. N'importe qui de bien disposé pourrait vivre parmi eux de la manière qui lui chante, mais il serait à la fin gagné par la monotonie. C'est ce que je montre avec le principal personnage blanc de mon récit. Le seul que j'ai croqué d'après un modèle vivant: le «châtelain » anglais qui m'a envoyé aux États-Unis. Je me souviens, quand mes premiers poèmes sont sortis, les femmes du marché m'arrêtaient sur le bord de la route et me demandaient de leur lire mes vers. Ce furent les lectures les plus heureuses de ma vie - je déteste lire en public. Je vous envoie quelques coupures supplémentaires. Je me souviens que vous m'avez parlé d'un article sur la boxe. Sparrow Robertson est le plus ancien écrivain de sport encore vivant, très juste envers les boxeurs noirs, il a connu les meilleurs d'entre eux et pourrait vous faire, dans son style unique, un article à leur propos.

II y a aussi une Martiniquaise, Paulette Nardal, 7 rue Hébert à Clamart, près de Paris, qui édite la Revue du monde noir ${ }^{\mathbf{2 7}}$. Je lui ai parlé de vous dans une lettre. Elle pourrait vous être utile. J'ignore s'il faut que je vous précise qu'il vaudrait mieux ne pas lui tenir un langage trop à gauche. 
J'ai trouvé que les socialistes, les communistes et les Noirs engagés dans le combat pour l'égalité sociale sont souvent plus sectaires que certains septembre 1932 bourgeois dans leurs conceptions intellectuelles et morales. Je souscris, (extraits) bien sûr, au programme économique du communisme, mais l'homme ne vit pas que de pain. Ma principale objection, à l'encontre des communistes, concerne leur attitude vis-à-vis de l'art et des artistes. Je ne crois pas à l'art prolétarien, ni à l'art bourgeois, ni à toute autre définition de l'art en termes vaguement sociaux. Pour moi l'art authentique est l'art qui, quelles que soient ses origines, raciales, nationales ou sociales, transcende son cadre initial et devient le bien de l'humanité dans son entier, et je me fiche bien des distinctions pédantes et des noms par lesquels on le nomme si tout cela ne sert pas à clairement l'identifier. Les communistes se sont lancés dans des diatribes à propos de l'art prolétarien et des artistes. Mais l'art véritable est bien trop rare pour être édicté par la politique. Car le communisme c'est de la politique, comme le socialisme, le républicanisme, le monarchisme - une science pour gouverner les gens et pour réglementer leur vie sociale et économique. L'artiste qui a des convictions communistes sur le plan politique n'en devrait pas moins être aussi libre de s'exprimer dans une société communiste que l'est son confrère bourgeois dans une société républicaine ou monarchiste. Je tiens pour erronée la théorie communiste de l'art prolétarien.

Elle ne vise qu'un art de propagande et si je veux bien croire à l'utilité de la propagande dans l'organisation politique, cela ne saurait valoir pour l'art. Ma conception de l'art est romantique. Je le salue partout où je le rencontre. Je ne m'attends pas à ce que les artistes soient des anges emplis de fraternité et d'amour pour l'humanité tout entière, même si je les apprécie d'autant plus s'ils possèdent ces qualités. 